International Journal of Medical Sciences

ISSN 1449-1907 www.medsci.org 2006 3(2):53-56

Review

\title{
Hepatitis C Virus (HCV) Infection and Hepatic Steatosis
}

\author{
Eugene J. Yoon, and Ke-Qin Hu \\ Division of Gastroenterology and Hepatology, University of California, Irvine Medical Center, CA 92868, USA
}

Corresponding address: Ke-Qin Hu, MD, Director of Hepatology Services and Associate Professor of Clinical Medicine, Division of Gastroenterology, Univ. of California, Irvine Medical Center, 101 The City Drive, Building 53, Suite 113, Orange, CA 92868, USA. Phone: 714-456-6745. Email: kqhu@uci.edu

Received: 2005.12.30; Accepted: 2006.02.16; Published: 2006.04.01

There are two discrete forms of steatosis that may be found in patients infected with hepatitis $\mathrm{C}$ virus (HCV). Metabolic steatosis can coexist with HCV, regardless of genotype, in patients with risk factors such as obesity, hyperlipidemia, and insulin resistance. The second form of hepatic steatosis in HCV patients is a result of the direct cytopathic effect of genotype 3 viral infections. There have been proposed mechanisms for this process but it remains elusive. Both categories of steatosis tend to hasten the progression of liver fibrosis and therefore prompt recognition and management should be initiated in patients with $\mathrm{HCV}$ and steatosis. The authors review the current understanding of the relationship between hepatitis $C$ infection and hepatic steatosis and discuss future research directions.

Key words: hepatitis C, obesity, metabolic syndrome, diabetes, steatosis, NAFLD, NASH

\section{Introduction}

The usual progression of liver disease in patients with hepatitis $\mathrm{C}(\mathrm{HCV})$ is a process of inflammation accompanied by periportal necrosis and fibrosis. The inflammation that results from the virus causes stimulation of stellate cells which ultimately leads to the deposition of collagen which leads to fibrosis progression within the liver. If this process is rapid and unhindered then the usual outcome is the development of cirrhosis which is the final irreversible stage characterized by parenchymal nodules with encircling fibrous septa. The hepatitis $C$ virus is not considered to directly injure the liver but it rather triggers an HCV-specific lymphoproliferation. Through profuse cytokine production and also a direct cytopathic effect, these T cells result in hepatocyte apoptosis. [1]

Many patients with chronic HCV are also noted to have a degree of steatosis present on their liver biopsies. Hepatic steatosis is defined as excessive lipid accumulation within the hepatocyte cytoplasm and has been more recently recognized as a significant cause for cirrhosis in the United States. [2] There are two forms of steatosis present in patients with hepatitis $C$, specifically metabolic steatosis and $\mathrm{HCV}$-induced steatosis. Metabolic steatosis is a process which occurs in the setting of obesity, hyperlipidemia, and insulin resistance. This form of steatosis is also similar to the type of fatty infiltration which occurs from excessive alcohol consumption. Metabolic steatosis is not triggered in anyway by the hepatitis $C$ virus however the combination of this form of steatosis and the presence of HCV has been associated with a more rapid progression of fibrosis. The other type of steatosis found in patients with HCV is fatty infiltration that is directly elicited by the virus. Though the precise mechanism is not well known, HCV-induced steatosis is recognized as the sole route for a direct cytopathic effect by the hepatitis $C$ virus. This review will focus on the two different forms of steatosis and its implications on the natural history of $\mathrm{HCV}$.

\section{Metabolic Steatosis}

The etiology for hepatic steatosis can be determined by the distribution and size of the lipid accumulation within the hepatocytes. Microvesicular steatosis that is seen in Reye's syndrome and Acute Fatty Liver Disease of Pregnancy occurs due to dysfunctions in $\beta$-oxidation of free fatty acids and this can result in acute liver failure. [3] On the other hand, macrovesicular steatosis is the histologic finding in patients with Non-alcoholic Fatty Liver Disease (NAFLD). NAFLD is characterized by gross macrovesicular fatty change with lobular or portal inflammation in the absence of a significant alcohol history. This disease is frequently under-recognized and is now identified as the most common cause of cryptogenic cirrhosis. [4] NAFLD occurs in the setting of obesity, hyperlipidemia, and diabetes all of which is now accepted under one syndrome called the Metabolic Syndrome.

The Metabolic Syndrome (also known as syndrome $\mathrm{X}$, the deadly quartet, the insulin resistance syndrome, and the obesity dyslipidemia syndrome) consists of abdominal obesity leading to insulin resistance, hypertension, and hypertriglyceridemia and is now recognized as the major predisposition to hepatic steatosis. [5-6] The most widely supported theory recognizes insulin resistance as the major mechanism in the pathogenesis of hepatic steatosis. [7-11] In an autopsy report from 1990, investigators found that of 22 patients with histologically confirmed fatty liver disease, 20 were also obese and had diabetes mellitus. [12]

The presumption that there is a causal relationship between the Metabolic Syndrome and disease progression in $\mathrm{HCV}$ makes sense given the connection between steatosis and necroinflammatory activity in patients with HCV. [13-14] Previous studies have indicated that obesity is a risk factor independent of elevated ALT levels which predict fibrosis progression. A study from Spain found that there is a larger proportion of fast progressors and a lower proportion of slow progressors in patients with body mass index (BMI) levels of greater than $30 \mathrm{~kg} / \mathrm{m}^{2}$. 
[15] Recognizing this risk factor may warrant weight loss in our obese patients just as much as alcohol abstinence in our patients with HCV. A small study of 19 patients showed that a weight loss of only $5.9 \mathrm{~kg}$ and a reduction in BMI by only $2 \mathrm{~kg} / \mathrm{m}^{2}$ may account for a significant reduction in both steatosis and fibrosis progression in patients with hepatitis C. [16] Thus, our more motivated patients with hepatitis $C$ absolutely deserve the extra means available for weight loss, not only for cardiovascular health but also to reduce the risk of fibrosis and ultimately cirrhosis.

Patients with the metabolic syndrome have some degree of insulin resistance. Diabetes was once thought to be an ailment secondary to the lack of insulin production but is now recognized as an illness of insulin resistance and resultant hyperinsulinemia. First line therapy now specifically targets insulin-mediated glucose utilization in the liver and peripheral tissue. [17-18] It has now been demonstrated that improvement occurs in both steatosis and the resultant inflammation by the use of insulin sensitizing agents. [19] Therefore, utilization of these medications are becoming more and more necessary in our patients with non-alcoholic steatohepatitis and certainly in our patients with $\mathrm{HCV}$ and metabolic steatosis.

\section{HCV-Induced Steatosis}

The presence of steatosis on liver biopsy in patients with hepatitis $C$ is more frequent when compared to other chronic liver diseases such as chronic hepatitis B and autoimmune hepatitis. [20] Steatosis is also 2.5 times more prevalent in patients with $\mathrm{HCV}$ when compared to the general population. [21] The macrovesicular steatosis present in patients with HCV is also distributed in the periportal areas rather than the centrilobular region which is more commonly seen in NAFLD. [22] This all infers that the hepatitis $\mathrm{C}$ virus may be directly inducing steatosis in these patients rather than simply being an unrelated finding.

It has been shown that HCV genotype 3 is independently associated with hepatocellular steatosis in patients with chronic hepatitis C. [23] Furthermore, the severity of steatosis in these patients is directly related to the burden of the HCV RNA load. This relationship between the $\mathrm{HCV}$ viral load and the magnitude of steatosis was not observed in other HCV genotypes. [24] It has also been noticed that the steatosis which was initially present in patients with genotype 3 infection resolves after a sustained virologic response is achieved through treatment with pegylated interferon- $\alpha$ and ribavirin. [25] Not only does the steatosis resolve with eradication of the virus but it also recurs if relapse transpires. All of these findings were only observed with the HCV genotype 3 virus and was not reproducible with other $\mathrm{HCV}$ genotypes. These findings all point to the ability of the HCV genotype 3 virus to directly induce steatosis, although the mechanism still remains elusive.

Thus, there seems to be two distinct forms of steatosis in patients with chronic hepatitis C. Metabolic steatosis generally occurs in all genotypes of $\mathrm{HCV}$ infections and likely worsens the progression of $\mathrm{HCV}$ induced fibrosis. Then there are those patients with genotype 3 infections who have a form of steatosis that is directly induced by the hepatitis $C$ virus and which also resolves with successful treatment. These two forms of steatosis can certainly coexist in patients with genotype 3 infections with other underlying metabolic diseases. In these patients, we would expect that the steatosis would only partially resolve with successful eradication of the virus and that the remaining fatty infiltration is a result of NAFLD.

\section{Mechanism of HCV-Induced Steatosis}

Most, if not all, studies to date have used HCV genotype 1 constructs in vitro, thus our current understanding of the process leading to lipid accumulation is limited. However, putative mechanisms exist where the presence of a particular viral component leads to lipid accumulation within the hepatocyte. HCV core protein has been studied at length in both cell cultures and in transgenic mice. Intracellular lipid buildup seems to occur when the HCV core protein is strongly expressed. [26] The core protein has been located at the surface of lipid droplets within the cytoplasm in cell cultures that are transfected with $\mathrm{HCV}$ while absent in control cells. [27]

$\mathrm{HCV}$ core protein may be interacting with apolipoprotein AII which is a major component of highdensity lipoproteins and this interaction may be causing hepatocellular steatosis. [28] Other proposed mechanisms suggest an interaction between the core protein and retinoid $X$ receptor a $(R \times R a)$ which is a transcriptional regulator that has many cellular functions, one of which is the metabolism of lipids. [29] Other theories propose that the core protein induces oxidative stress within the mitochondria which leads to or contributes to lipid accumulation. [30] This notion was formulated due to the association between hepatic steatosis in transgenic mice and the presence of increased lipid peroxidation. [31] All of these premises point to a complex interaction between the HCV core protein and other components of the hepatocyte which ultimately contributes to the onset of steatosis. Though the exact mechanism remains elusive, it seems firmly established that the hepatitis $C$ virus, in and of itself, can directly induce cytoplasmic lipid accumulation. Further studies examining the genotype 3 virus are warranted to further recognize the process involved in $\mathrm{HCV}$-induced steatosis.

\section{Disease Progression}

It is difficult to ascertain whether $\mathrm{HCV}$-induced steatosis and metabolic steatosis contribute equally to the overall disease progression in patients with hepatitis $C$. As discussed before, there have been many studies which have demonstrated a relationship between the severity of steatosis and the extent of fibrosis on liver biopsy specimens. However, the majority of studies to date have not separated the two types of steatosis when examining its affects on fibrosis progression in $\mathrm{HCV}$ patients. Nonetheless, it has been generally accepted that either steatosis by itself aggravates fibrosis or the factors that are causing steatosis may be aggravating fibrosis. [32] There has also been some suggestion that the two forms of steatosis may act synergistically to trigger severe advancement of fibrosis in patients with genotype 3 infections. [32] Overall, steatosis, whether metabolic or $\mathrm{HCV}$ induced, worsens the sequence of events leading to advanced fibrosis in patients with HCV and needs to be addressed when managing our patients with HCV. Ultimately, further studies are warranted to distinguish viral steatosis between metabolic steatosis and both of their effects on fibrosis progression in patients with $\mathrm{HCV}$. 


\section{Conclusion}

All in all, there is a clinically significant relationship between HCV infection and hepatic steatosis. Two forms of steatosis exist in our patients with HCV. The first type of steatosis occurs secondary to metabolic factors namely alcohol use or the metabolic syndrome. This form of steatosis is not initiated by the hepatitis $C$ virus however it can very well increase the progression of fibrosis ultimately towards cirrhosis. The other form of steatosis occurs as a direct result of the HCV genotype 3 virus through complex interactions between the HCV core protein and the hepatocyte, the knowledge of which remains to be fully unravelled. This type of steatosis also very well causes more rapid progression of disease. It is important to recognize the category of steatosis present in our patients with $\mathrm{HCV}$ in order to properly treat them. Those with metabolic steatosis warrant strict attention to weight loss and countering the effects of insulin resistance while focus on $\mathrm{HCV}$ eradication can be given to those with $\mathrm{HCV}$-induced steatosis.

\section{Research Direction}

It is well established that there is an association between HCV and steatosis and the mechanisms behind this relationship are currently being unravelled. In order to fully understand the intricate molecular processes involved in HCV genotype-3 induced hepatocellular steatosis, future studies need to analyze viral and metabolic steatosis as distinct groups. Research should also continue to focus on therapies for insulin resistance and steatosis-induced fibrosis in chronic hepatitis C. In order to devise specific therapies for $\mathrm{HCV}$-induced steatosis, further investigation needs to be done on the complex derangements in lipid metabolism with focus on the genotype 3 virus.

\section{Conflict of interest} exists.

The author has declared that no conflict of interest

\section{References}

1. Poynard T, Bedossa P, Opolon P. Natural history of liver fobrosis progression in patients with chronic hepatitis C. The OBSVIRC, METAVIR, CLINIVIR, and DOSVIRC groups. Lancet. 1997;349(9055):825-32.

2. Brunt EM. Nonalcoholic steatohepatitis: Definition and pathology. Semin Liver Dis 2001;21:3-16.

3. Burt AD, Mutton A, Day CP. Diagnosis and interpretation of steatosisi and steatohepatitis. Semin Diagn Pathol 1998;15:246-58.

4. Clark JM, Diehl AM. Nonalcoholic fatty liver disease: an underrecognized cause of cryptogenic cirrhosis. JAMA. 2003;289(22):3000-4.

5. Scheen AJ, Luyckx FH. Nonalcoholic steatohepatitis and insulin resistance: interface between gastroenterologists and endocrinologists. Acta Clin Belg. 2003;58(2):81-91.

6. Marchesini G., Bugianesi E, Forlani G, et al. Nonalcoholic fatty liver, steatohepatitis, and the metabolic syndrome. Hepatology 2003; 37:917.

7. Sanyal AJ, Campbell-Sargent C, Mirshahi F, et al. Nonalcoholic steatohepatitis: Association of insulin resistance and mitochondrial abnormalities. Gastroenterology 2001; 120:1183.

8. Chitturi S, Abeygunasekera S, Farrell GC, et al. NASH and insulin resistance: Insulin hypersecretion and specific association with the insulin resistance syndrome. Hepatology 2002; 35:373.

9. Willner IR, Waters B, Patil SR, et al. Ninety patients with nonalcoholic steatohepatitis: Insulin resistance, familial tendency, and severity of disease. Am J Gastroenterol 2001; 96:2957.
10. Pagano G, Pacini G, Musso G, et al. Nonalcoholic steatohepatitis, insulin resistance, and metabolic syndrome: Further evidence for an etiologic association. Hepatology 2002; 35:367.

11. Marchesini G, Brizi M, Morselli-Labate AM, et al. Association of nonalcoholic fatty liver disease with insulin resistance. Am J Med 1999; 107:450.

12. Wanless IR, Lentz JS. Fatty liver hepatitis and obesity: An autopsy study and analysis of risk factors. Hepatology 1990; 12:1106.

13. Clouston AD, Jonsson JR, Purdie DM, Macdonald GA, Pandeya N, Shorthouse C, et al. Steatosis and chronic hepatitis C: Analysis of fibrosis and stellate cell activation. J Hepatol 2001;34:314-20.

14. Adinolfi LE, Gambardella M, Andreana A, Tripodi MF, Utili R, Ruggiero G. Steatosis accelerates the progression of liver damage of chronic hepatitis $C$ patients and correlates with specific $\mathrm{HCV}$ genotype and visceral obesity. Hepatology 2001;33:1358-64.

15. Ortiz V, Berenguer M, Rayon JM, Carrasco D, Berenguer J. Contribution of obesity to hepatits C-related fibrosis progression. Am J Gastroenterol 2002;97:2408-2414.

16. Hickman IJ, Clouston AD, Macdonald GA, Purdie DM, Prins JB, Ash S, et al. Effect of weight reduction on liver histology and biochemistry in patients with chronic hepatitis C. Gut 2002;51:89-94.

17. Bailey CJ, Turner RC. Metformin. N Engl J Med 1996; 334:574.

18. Nolan JJ, Ludvik B, Beerdsen P, Joyce M, Olefsky J. Improvement of glucose tolerance and insulin resistance in obese subjects treated with trogliazone. N Engl J Med 1994; 331:1188.

19. Neuschwander-Tetri BA, Brunt EM, Wehmeier KR, et al. Improved nonalcoholic steatohepatitis after 48 weeks of treatment with the PPAR-gamma ligand rosiglitazone. Hepatology. 2003 Oct;38(4):1008-17.

20. Hwang SJ, Luo JC, Chu CW, et al. Hepatic steatosis in chronic hepatitis C virus infection: Prevalence and clinical correlation. J Gastroenterol Hepatol 2001;16:190-5.

21. Lonardo A, Adinolfi LE, Loria P, et al. Steatopsis and hepatitis C virus: Mechanisms and significance for hepatic and extrahepatic disease. Gastroenterology 2004;126:586-97.

22. Zaitoun AM, Al Mardini H, Awad S, et al. Quantitative assessment of fibrosis and steatosis in liver biopsies from patients with chronic hepatitis C. J Clin Pathol 2001;54:461-5.

23. Rubbia-Brandt L, Quadri R, Abid K, et al. Hepatocyte steatosis is a cytopathic effect of hepatitis C virus genotype 3. J Hepatol 2000;33:106-15.

24. Hezode C, Roudot-Thoraval F, Zafrani ES, et al. Different mechanisms of steatosis in hepatitis $C$ virus genotypes 1 and 3 infections. J Viral Hepat 2004;11:455-8.

25. Patton HM, Patel K, Behling C, et al. The impact of steatosis on disease progression and early and sustained treatment response in chronic hepatitis C patients. J Hepatol 2004;40:484-90.

26. Barba G, Harper F, Harada T, et al. Hepatitis C virus core protein shows a cytoplasmic localization and associates to cellular lipied storage droplets. Proc Natl Acad Sci U S A 1997;94:1200-5.

27. Moradpour D, Englert C, Wakita T, et al. Characterization of cell lines allowing tightly regulated expression of hepatitis $C$ virus core protein. Virology 1996;222:51-63.

28. Sabile A, Perlemuter G, Bono F, et al. Hepatitis C virus core protein binds to apolipoprotein AII and its secretion is modulated by fibrates. Hepatology 1999;30:1064-76.

29. Tsutsumi T, Suzuki T, Shimoike T, et al. Interaction of hepatitis C virus core protein with retinoid $\mathrm{X}$ receptor alpha modulates its transcriptional activity. Hepatology 2002;35:937-46.

30. Okuda M, Li K, Beard MR, et al. Mitochondrial injury, oxidative stress, and antioxidant gene expression are induced by hepatitis $\mathrm{C}$ virus core protein. Gastroenterology 2002;122:366-75.

31. Lerat $\mathrm{H}$, Handa M, Beard MR, et al. Steatosis and liver cancer in transgenic mice expressing the structural and nonstructural proteins of hepatitis C virus. Gastroenterology 2002;122:352-65.

32. Castera L, Chouteau P, Hezode C, et al. Hepatitis C Virus-Induced Hepatocellular Steatosis. Am J Gastroenterol 2005;100:711-15.

\section{Author biography}

Eugene J. Yoon, MD, is a fellow in the Department of Medicine, Division of Gastroenterology and Hepatology, University of California, Irvine, California, USA. 
Ke-Qin Hu, MD, is the Director of Hepatolgy Services and Associate Professor of Clinical Medicine, Division of Gastroenterology, University of California, Irvine, California, USA. His current research interests include the natural history and management of hepatitis $B$ and $C$ and chemoprevention of hepatocellular carcinoma. 